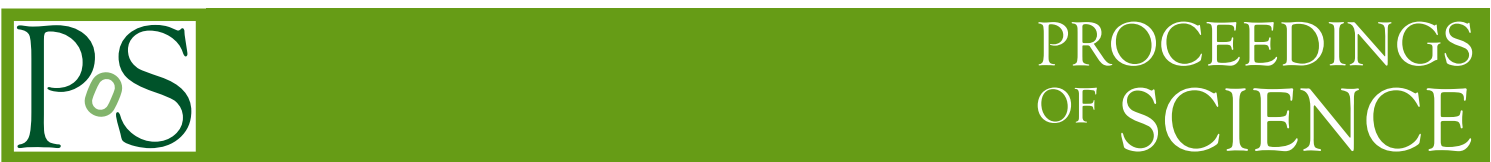

\title{
Scale setting in lattice QCD
}

\author{
Rainer Sommer \\ NIC@DESY, Platanenallee 6, 15738 Zeuthen, Germany \\ E-mail: Rainer.Sommeredesy.de
}

The principles of scale setting in lattice QCD as well as the advantages and disadvantages of various commonly used scales are discussed. After listing criteria for good scales, I concentrate on the main presently used ones with an emphasis on scales derived from the Yang-Mills gradient flow. For these I discuss discretisation errors, statistical precision and mass effects. A short review on numerical results also brings me to an unpleasant disagreement which remains to be explained.

31st International Symposium on Lattice Field Theory - LATTICE 2013

July 29 - August 3, 2013

Mainz, Germany 


\section{Introduction}

Presumably the most natural scale for low energy QCD is the mass of the proton, $m_{\mathrm{p}}$. It is very well knwown. Alternatively, as a theorist, one might like the pseudo scalar decay constant in the chiral limit, called $f$. It sets the scale for interactions of the very low energy description of QCD. Indeed, let us consider for a little while just QCD in the chiral limit, in order to simplify the discussion. In this limit, ratios of pairs of these scales or any other observables of dimension mass, $m_{i}$, are predictions of the theory, but not the scale itself. The latter, and only it, has to be taken from experiment. In lattice QCD this manifests itself in the fact that dimensionless ratios,

$$
\mathscr{R}_{i}=m_{i} / m_{\mathrm{p}}
$$

can be computed and have a continuum limit. Taking $m_{\mathrm{p}}$ as a reference here is what we call scale setting. Equivalently, a lattice computation determines dimensionless quantities $M_{i}=m_{i} a, M_{\mathrm{p}}=$ $m_{\mathrm{p}} a$ as a function of the bare coupling $g_{0}$. The prediction for the physical, dimensionful quantity, $m_{i}$ is then obtained by

$$
m_{i}=\mathscr{R}_{i} m_{\mathrm{p}}, \quad \mathscr{R}_{i}=\lim _{M_{\mathrm{p}} \rightarrow 0} \frac{M_{i}}{M_{\mathrm{p}}}=\lim _{g_{0} \rightarrow 0} \frac{M_{i}\left(g_{0}\right)}{M_{\mathrm{p}}\left(g_{0}\right)} .
$$

In lattice slang, scale setting usually is refered to the equivalent point of view of determining the lattice spacing $a$ at a given $g_{0}$ from the specific quantity $m_{\mathrm{p}}$,

$$
a_{\mathrm{p}}\left(g_{0}\right)=\frac{M_{\mathrm{p}}\left(g_{0}\right)}{m_{\mathrm{p}}},
$$

with the purpose of then defining any other dimensionful quantity

$$
m_{i}^{\text {lat }}\left(g_{0}\right)=\frac{M_{i}\left(g_{0}\right)}{a_{\mathrm{p}}\left(g_{0}\right)}
$$

Clearly $m_{i}^{\text {lat }}\left(g_{0}\right)$ has a continuum limit and at finite $g_{0}$ (finite $a$ ) it has lattice artifacts (deviations from this limit) which depend also on the choice of scale, $m_{\mathrm{p}}$.

In practise, we also approximate QCD by an effective theory where heavy quarks are removed (one often says integrated out). How good this approximation is also depends on the choice of the scale as is apparent from the previous discussion.

\subsection{Scale setting is important}

Why is scale setting discussed in a plenary talk? Both in planning simulations and in the analysis of the results, the scale (as explained above, whether I say the scale or the lattice spacing is just the same) is usually very important. When quark masses are neglected, an error made in the scale, systematic or statistical, propagates linearly into a hadron mass.

Now thinking about the real theory, the one with quark masses, the scale already enters decisively into fixing the bare quark masses in the Lagarangian, namely in planning the simulations. Usually we do this by adjusting the pseudo scalar meson masses to their physical values, since those are most sensitive to quark masses. The scale is needed for that. It is very important to perform simulations at or close to the right quark mass, or on a given desired trajectory running through the physical point, in the parameter space given by the quark masses. For mass-degenerate 
u,d quarks, a trajectory may be given by fixed (physical or bare PCAC) strange (and ultimately charm quark) masses or for example by a fixed trace of the quark mass matrix, $\operatorname{tr} m_{\text {quark }}=$ const. In the past several collaborations missed the proper trajectory by more than what they would have liked to.

Furthermore, the scale enters where a momentum in a form factor is fixed or into the overall size of the lattice. It is very useful to know the scale beforehand.

We should also draw lessons from recent years. In a few cases, challenging new computations have been carried out, relying on the scale from earlier work. Later it turned out that there was an inaccuracy in the scale, which significantly influenced the result. Examples are changes in the decay constant $f_{\mathrm{D}_{\mathrm{s}}}$ of the HPQCD collaboration due to a reanalysis of $r_{1}[1,2]$ and one that the ALPHA collaboration made, namely a roughly $20 \%$ change in the two-flavour $\Lambda$-parameter after we had computed $r_{0} / a$ rather than taking it from the literature [3].

All of this calls for care in the selection and computation of the scale.

\subsection{What is a good scale?}

Unfortunately, the proton mass is not easily determined with good precision in lattice QCD computations due to a large noise/signal ratio in the proton correlation function, see Sect. 2.1. The chiral scale $f$ is related to experiments only through the chiral perturbation theory expansion. Consequently it is not common to use these observables to set the scale. In general we should search for a quantity which

(P1) is computable with a low numerical effort,

(P2) has a good statistical precision,

(P3) has small systematic uncertainties and

(P4) has a weak quark mass dependence.

The first two properties are self explanatory, but the others require some details. By a good systematic precision we mean first of all that the systematic error in the determination of the numbers $M_{i}=a m_{i}$ for given bare coupling and quark masses is small. For example such a systematic error may come from finite size effects or the contamination by excited states. A second systematic uncertainty is the discretisation error. This is not easily judged. We will comment on it as we go along. Concerning the quark masses, it is of course useful to have a scale which depends weakly on them as this makes the tuning of the quark masses rather independent from the scale and, when the simulations are not at the physical point, the extrapolation/interpolation to it is easier. Note that the sensitivity to quark masses also depends on the trajectory one chooses to reach the physical point. For example the mass of the Omega-baryon has a weak dependence on the light quark masses when the strange quark mass is fixed, but not when one is on the trajectory tr $m_{\text {quark }}=$ const. On such a trajectory an appropriate average baryon mass has a weak quark-mass dependence, see e.g. [4].

For later use I define a measure for the quark mass dependence,

$$
S_{Q}^{\mathrm{m}}=\frac{\left.Q\right|_{m_{\pi}=500 \mathrm{Mev}}-\left.Q\right|_{m_{\pi}=130 \mathrm{MeV}}}{\left.Q\right|_{m_{\pi}=130 \mathrm{MeV}}},
$$


where $Q$ labels different scales, e.g. $Q=m_{\mathrm{p}}$, and we assume that we are on a trajectory with strange and charm (bare, PCAC) mass fixed. Selfconsistently, $\mathrm{MeV}$ come from the scale $Q$. Since there is usually a rather linear dependence of scales on $m_{\pi}^{2}$, one has $S_{Q}^{\mathrm{m}} \approx \frac{0.23 \mathrm{GeV}^{2}}{\left.Q\right|_{m \pi}=130 \mathrm{MeV}} \frac{\partial Q}{m_{\pi}^{2}}$.

The statistical precision is determined both by the integrated autocorrelation time and by the variance. ${ }^{1}$ These depend on the update algorithm and on the chosen estimators, respectively. E.g. in the simplest case, using or not-using or stochastically-using translation invariance are different estimators of a correlation function.

After this preparation, let me come to a discussion of a few scales which are in frequent use or newly proposed. I differentiate between phenomenological scales and theory scales. The former are related to physical observables through a minimum amount of theory, while the latter are constructed to be well computable in lattice QCD but their values can (at present) only be computed in lattice QCD using a phenomenological scale as an input. Thus a phenomenological scale is needed in any case. The distinction between one category and the other is not sharp. For example I place $r_{0}$ [6] with the theory scales, although (vague) phenomenological considerations led to the prediction $r_{0} \approx 0.49 \mathrm{fm}$ which is not far from our present knowledge.

\section{Phenomenological scales}

\subsection{The mass of the $\Omega$-baryon.}

The relative errors $R_{N / S}$ of baryon correlation functions grow at large time, $x_{0}$, as $[7,8]^{2}$

$$
\begin{aligned}
& R_{N / S}^{\mathrm{p}} \stackrel{x_{0} \text { large }}{\sim} K_{\mathrm{p}} \exp \left(\left(m_{\mathrm{p}}-\frac{3}{2} m_{\pi}\right) x_{0}\right) \approx \exp \left(x_{0} / 0.27 \mathrm{fm}\right), \\
& R_{N / S}^{\Omega} \stackrel{x_{0} \text { large }}{\sim} K_{\Omega} \exp \left(\left(m_{\Omega}-\frac{3}{2} m_{\eta_{s}}\right) x_{0}\right) \approx \exp \left(x_{0} / 0.31 \mathrm{fm}\right),
\end{aligned}
$$

using $m_{\eta_{s}}^{2} \approx 2 m_{\mathrm{K}}^{2}-m_{\pi}^{2}$. The difference in the two formulae is one reason, why there is hardly a computation where the scale is taken from the proton mass, but a few groups like to set it through $m_{\Omega}$. The numerical values inserted for the masses are taken from the PDG and refers to the physical point. Unfortunately it is not common any more to publish a plot of the effective mass to illustrate the quality of the plateau. In Fig. 1 I show one using data from CLS ensemble N6, with $a=$ $0.045 \mathrm{fm}, m_{\pi}=340 \mathrm{MeV}$ on a $48^{3} 96$ lattice (for details on these ensembles see [9]), which has a statistics of about $8000 \mathrm{MDU} \approx 40 \tau_{\mathrm{exp}}$. One observes smaller statistical errors compared to the proton, but in fact the relation to the large time asymptotics, eq. (2.1), is not entirely obvious. The plateaux for $m_{\Omega}, m_{\mathrm{p}}$ appear to start around $0.8 \mathrm{fm} \approx 1.6 r_{0}$ or so. Somewhat earlier plateaux (at $\approx 0.6 \mathrm{fm})$ are visible in $[10,11]$, where correlation functions with different smeared interpolating fields are considered which use gauge fixing.

A second advantage of $m_{\Omega}$ is the weak dependence on the light quark mass, if the strange mass is fixed. Reversely, it depends much more on the strange mass than other common choices.

\footnotetext{
${ }^{1}$ For the definition of variance and autocorrelation times for derived observables (such as masses extracted from a correlation function, the primary observable) see [5].

${ }^{2}$ Note that the arguments of $[7,8]$ refer to the square root of the variance of the correlation functions; a $x_{0^{-}}$ dependence of the autocorrelation time is negelceted.
} 


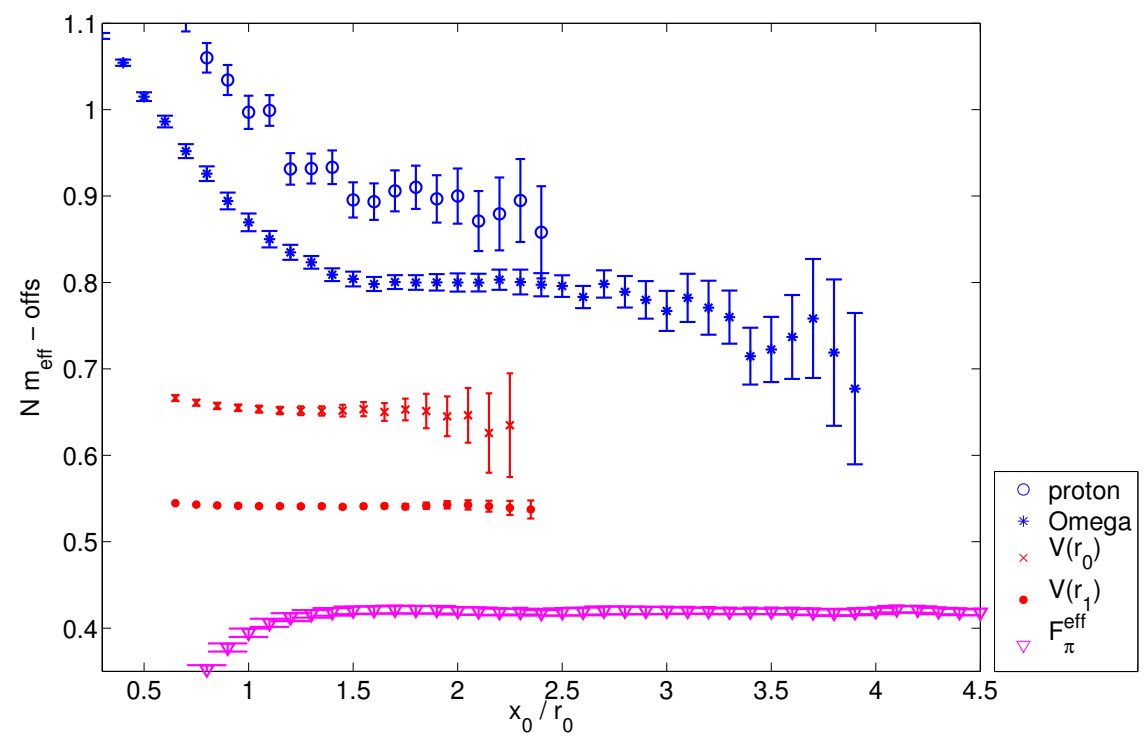

Figure 1: Effective masses for $m_{\mathrm{p}}$ [12], $m_{\Omega}$ [13], $V\left(\approx r_{0}\right), V\left(\approx r_{1}\right)$ [14] and $f_{\pi}$ [9] on CLS ensemble N6 (see [9]). All effective "masses" have been scaled such that the errors in the graph reflect directly the errors of the determined scales. They have been shifted vertically.

\subsection{Pseudo scalar decay constants $f_{\pi}, f_{\mathrm{K}}$}

Pseudo scalar decay constants have been popular with several collaborations. In particular they have been used to perform the primary scale setting and then determine the values of the theory scales $r_{0}, r_{1}, t_{0}, w_{0}$ in terms of these. A drawback of decay constants is that experimentally they are determined from weak processes. The $\pi \rightarrow \ell v$ decay rate yields the product $V_{\mathrm{ud}} f_{\pi}$ and the decay rate $K \rightarrow \ell v$ is given by $V_{\text {us }} f_{\mathrm{K}}$. Thus the precision we can achieve for $f_{\pi}, f_{\mathrm{K}}$ is limited by our knowledge of the CKM matrix elements $V_{\mathrm{ud}}$ and $V_{\mathrm{us}}$, where in particular one needs to assume a dominance by the standard model processes and a correct determination of the matrix elements of other processes from which $V_{\mathrm{ud}}$ and $V_{\mathrm{us}}$ are derived.

On the other hand, a clear advantage is the small and almost $x_{0}$-independent variance of the pseudo-scalar correlators, leading to long plateaux, see Fig. 1. I will come back to the importance of long plateaux in the conclusions.

\subsubsection{Autocorrelations}

I would like to emphasise a further feature of the error analysis of the decay constants. We have learnt in recent years that one has to be careful concerning the contribution of slow modes of the Markov matrix to the autocorrelation function $\rho_{O}\left(t_{\mathrm{MC}}\right)$ of an observable $O$. Such modes contribute as one (or several) slowly decaying exponentials $A \exp \left(-t_{\mathrm{MC}} / \tau_{\exp }\right)$. Pseudo scalar correlators, at least those computed with a noisy estimator for the source-timeslice spatial average of the correlation function, show a rather small amplitude $A$ of these potentially dangerous terms. I show an example for the aforementioned N6 ensemble in Fig. 2. This ensemble has a relatively long exponential autocorrelation time $\tau_{\exp } \approx 200$ and thus even a small amplitude of $A \sim 1 / 20$ leads to a contribution of order 10 in the integrated autocorrelation time. Still this is a small $\approx 50 \%$ 
part of $\tau_{\text {int }}$. Most of the relevant (and accessible) part of the autocorrelation function is dominated by shorter time scales of the MC process.

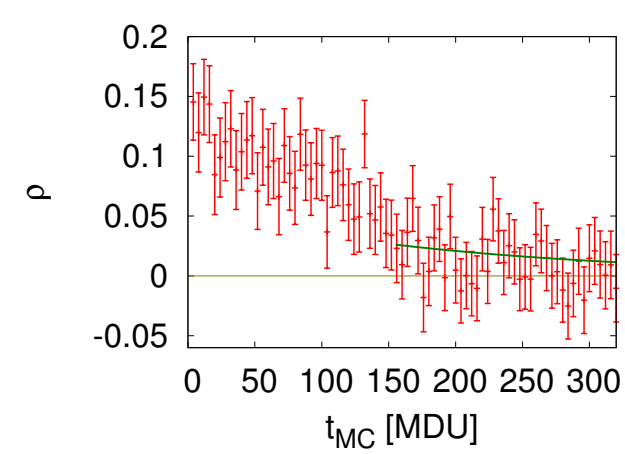

Figure 2: Normalised $(\rho(0)=1)$ autocorrelation function of $f_{\pi}$ on CLS ensemble N6 [9]. We show the estimate of a contribution of a tail, following the analysis of [15].

Note that the discussion of autocorrelation issues in QCD simulations is only semi-quantitative, in particular our estimates of the tails according to [15]. However, this is hardly avoidable given our still limited abilities to generate long $\mathrm{MC}$ ensembles. A consideration of a possible tail and some estimate of the influence of a tail is important exactly because statistics is not large enough to just sum up $\rho\left(t_{\mathrm{MC}}\right)$ to sufficiently large $t_{\mathrm{MC}}$.

Despite these problems, my message here is that autocorrelations are a minor problem for the decay constants, as long as one is not in the desastrous region of parameters, which as far as we know starts rather close to $a \approx 0.045 \mathrm{fm} \mathrm{[15-17]} \mathrm{for} \mathrm{periodic}$ boundary conditions.

\subsection{A scale from the vector correlator}

Over the years an impressive phenomenological knowledge about the vector-meson spectral function has been built up, largely due to the efforts to provide a precise number for the hadronic vacuum polarisation contribution to the magnetic moment of the muon, $g_{\mu}$ [18]. David Bernecker and Harvey Meyer recently proposed to build on this knowledge and predict the isovector Euclidean time-slice correlator $C_{\mathrm{V}}\left(x_{0}\right)$ from phenomenology [19]. The scale $\tau_{1}$ defined through $[19,20]$

$$
-\left.x_{0} \frac{\mathrm{d}}{\mathrm{d} x_{0}} \log C_{\mathrm{V}}\left(x_{0}\right)\right|_{x_{0}=\tau_{1}}=3.25
$$

can then be evaluated from phenomenology to be $\approx 0.73 \mathrm{fm}$ in $N_{\mathrm{f}}=2 \mathrm{QCD}$. Note that the "hidden strangeness" contributions are removed from the experimental value in order to arrive at a prediction more closely related to $N_{\mathrm{f}}=2$ QCD. Such a step is obviously not done/possible when one fixes the scale through $m_{\Omega}$ or $f_{\pi}$. The particular choice of 3.25 on the right hand side is a compromise between a large enough $x_{0}$ in $\frac{\mathrm{d}}{\mathrm{d} x_{0}} \log C_{\mathrm{V}}\left(x_{0}\right)$ beyond the perturbatively dominated regime with $\frac{\mathrm{d}}{\mathrm{d} x_{0}} \log C_{\mathrm{V}}\left(x_{0}\right) \sim 1 / x_{0}$ and to have a good statistical precision. Also finite size effecs grow with $x_{0}$.

An advantage of $\tau_{1}$ compared to hadron masses is that it is defined directly at finite $x_{0}$. But the necessary phenomenology is not entirely straight forward. In particular one has to separate the different isospin contributions in the experimental $e^{+} e^{-} \rightarrow$ hadrons cross section in order to obtain the isovector spectral function or one has to use the full electromagnetic current which requires quark-line disconnected contibutions. The method is presently being developed and tested [20].

\section{Theory scales}

Already the phenomenology needed for $\tau_{1}$ is somewhat involved. We now turn to scales defined in terms of the static quark potential. Even though a prediction of $r_{0} \approx 0.49 \mathrm{fm}$ based 


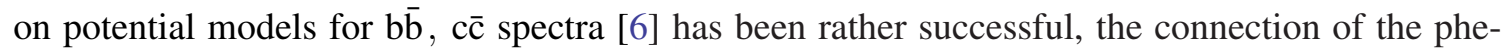
nomenological potentials to the static potential $V(r)$ has never become truely quantitative. I therefore consider both $r_{1}$ and $r_{0}$ as theory scales.

$3.1 r_{0}, r_{1}$

Their definition is [6]

$$
\left.r^{2} F(r)\right|_{r=r_{c}}=c, \quad r_{0} \equiv r_{1.65} .
$$

The original motivation for the definition of $r_{c}$ was an improvement over the string tension, which was used extensively as a reference scale in the pure gauge theory. The string tension requires a double limit of large time extent of a Wilson loop at fixed $r$ and then the limit of large $r$. The scales $r_{c}$ "only" require large time extent to extract the ground state potential.

Let me discuss a few properties. The force is given by the derivative of the potential. When a proper lattice derivative is used and and the (light-quark and gluon) action is $\mathrm{O}(a)$ improved, the force is $\mathrm{O}(a)$ improved. This property follows[21] from the automatic $\mathrm{O}(a)$ improvement of static quark actions [22]. In fact, different static actions with a (moderate!) smearing of the gauge field in the static action can be used $[23,24]$. In practise, apart from unsmeared links, corresponding to the Eichten-Hill action for the static quarks, mostly the HYP2 action [24] has been adopted. The reason is as follows. The relative errors of Wilson loops grow as

$$
R_{N / S}^{\mathrm{W}} \stackrel{T \text { large }}{\sim} K_{\mathrm{W}}(r) \exp \left(\left[\frac{e_{1} g_{0}^{2}+\ldots}{a}+\varepsilon(r)\right] T\right),
$$

where the mass scale in the exponent diverges in the continuum limit while $\varepsilon(r)$ is finite. The divergent term is the self energy of the static quark or, in a different language, the perimiter term in the Wilson loop. The coefficient $e_{1}$ depends on the static quark action and the HYP2 variant has been constructed such that it is rather small. One should keep in mind that different static actions mean different discretisation effects and hence different values for $r_{0}, r_{1}$ at finite lattice spacing.

The divergence in the exponent of eq. (3.2) will eventually become a serious problem when the continuum limit is approached more and more, but at present lattice spacings, the generalised eigenvalue method $[25,26]$ combined with smearing of the spatial parallel transporter still allows for sub-percent precision [27]. As shown in Fig. 1 this method achieves a very early plateau in $V(r)$. On the other hand, the MILC collaboration adopted $r_{1}$ as their standard [28] since at smaller $r$ the excited state corrections to the ground state decay much faster with $T$, allowing for smaller $T$ and associated smaller errors, see Fig. 1 . Of course, smaller $r / a$ comes with the price of generically larger $a^{2}$ effects. To a certain extent, kinematical $a^{2}$ effects can be supressed by a good definition of the discretised force [6] or by a phenomenological subtraction of $a^{2}$ effects from the potential [28].

Contributions of slow modes to the autocorrelation function of $r_{0}, r_{1}$ are less relevant than for $f_{\pi}, f_{\mathrm{K}}$, simply due to the relatively large variance of the Wilson loops.

\subsection{Scales derived from the gradient flow}

\subsubsection{Gradient flow and definition of $t_{0}, w_{0}$.}

The gradient flow is discussed at this conference by Martin Lüscher [29]. Here we only need the Yang-Mills flow. In continuum notation, one introduces a gauge field $B_{\mu}(x, t)$ which depends 
in addition to the space-time coordinates $x$ on a flow time $t$ and coincides with the quantum gauge field $A_{\mu}(x)$ at $t=0$. At positive flow-time, $B_{\mu}$ is defined through the flow equation [30]

$$
\frac{\mathrm{d}}{\mathrm{dt}} B_{\mu}(x, t)=D_{\nu} G_{v \mu}(x, t)=-\frac{\delta S_{\mathrm{YM}}[B]}{\delta B_{\mu}(x, t)}, \quad B_{\mu}(x, 0)=A_{\mu}(x)
$$

where $D_{v}$ is the covariant derivative in terms of the gauge field $B_{\mu}$ and $G_{\mu \nu}=\left[D_{\mu}, D_{v}\right]$. As a solution of eq. (3.3), $B_{\mu}$ is just a functional of the (true, quantum) gauge field. At lowest order in the weak coupling expansion, the flow equation becomes the heat equation with solution

$$
B_{\mu}(x, t)=\int \mathrm{d}^{4} y(4 \pi t)^{-2} \mathrm{e}^{-(x-y)^{2} /(4 t)} A_{\mu}(y)+\mathrm{O}\left(g_{0}^{2}\right) .
$$

We see that the gauge field has been smoothed over a radius of $\sqrt{8 t}$. For $t>0$, correlation functions of this smooth field are finite at any Euclidean distance [31], in particular

$$
\mathscr{E}(t)=t^{2}\langle E(x, t)\rangle, \quad E(x, t) \equiv-\frac{1}{2} \operatorname{tr} G_{\mu v}(x, t) G_{\mu v}(x, t)
$$

needs no renormalisation beyond the one of gauge coupling and quark masses. Thus, for example in $N_{\mathrm{f}}=2 \mathrm{QCD}, f\left(t / r_{0}^{2}, m_{\pi}^{2} r_{0}^{2}\right)=\mathscr{E}(t)$ is a finite function. The proof of finiteness to all orders of perturbation theory uses a formulation in terms of a 5-dimensional theory, adding the extra dimension $t$ (with dimension length ${ }^{2}$ ) and a boundary $t=0$ [31]. This 5-d formulation is also essential to perform a Symanzik analysis of cutoff effects [32] which I turn to in the following section.

First I define the scales [30, 33]

$$
\begin{aligned}
& t_{0}: \\
& w_{0}: t_{0}\left(t_{0}\right)=0.3, \\
& w_{0}^{2} \mathscr{E}^{\prime}\left(w_{0}^{2}\right)=0.3,
\end{aligned}
$$

where $\mathscr{E}^{\prime}(t)=\frac{\mathrm{d}}{\mathrm{d} t} \mathscr{E}(t)$. These are truely theory scales; we need to determine their values in terms of a phenomenological scale by a lattice QCD computation, performing the continuum limit.

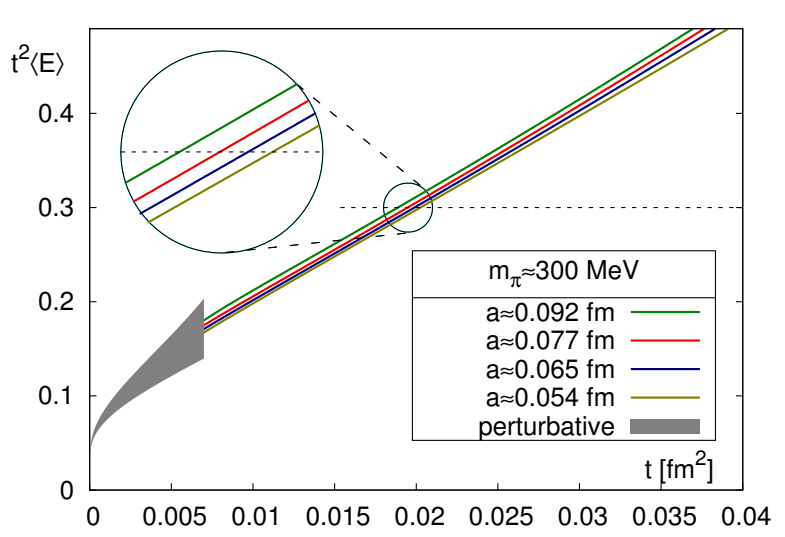

Figure 3: $N_{\mathrm{f}}=2+1$ results for $\mathscr{E}(t)$ from the BMW collaboration. Graph from [33].

lattice spacing effects compared to $t_{0}$. One should bear in mind that parallel lines in Fig. 3 turn to crossing ones, when the scale is set in a different way which has non-negligible $\mathrm{O}\left(a^{2}\right)$ cutoff effects relative to $m_{\Omega}$. We turn to a discussion of cutoff effects specific to the gradient flow. 


\subsubsection{Cutoff effects}

I start with some heuristics. Expressing quantities as integrals in momentum space, those dominated by small momenta have smaller cutoff effects than those dominated by large momenta, at least as long as no accidental cancellations take place. Fourier transforming eq. (3.4) yields a gaussian damping of large momenta and at lowest order of perturbation theory, in the continuum, we obtain

$$
\begin{aligned}
\mathscr{E}(t) & \sim g_{0}^{2} t^{2} \int_{0}^{\infty} p^{3} \mathrm{e}^{-2 t p^{2}} \mathrm{~d} p+\mathrm{O}\left(g_{0}^{4}\right) \\
t \mathscr{E}^{\prime}(t) & \sim g_{0}^{2} t^{2} \int_{0}^{\infty} p^{3}\left(1-t p^{2}\right) \mathrm{e}^{-2 t p^{2}} \mathrm{~d} p+\mathrm{O}\left(g_{0}^{4}\right) .
\end{aligned}
$$

The integrand of eq. (3.8) is peaked around $p \approx t^{-1 / 2}$ while in eq. (3.9) it has two peaks below and above that region whose integrals cancel. The essential observation is the damping of large momenta which renders $\mathscr{E}$ finite and supresses cutoff effects.

However, we note that $t_{0} \approx(0.15 \mathrm{fm})^{2}$ in QCD. Thus with a lattice spacing of $a \approx 0.1 \mathrm{fm}$, the dominating momenta have $a p \approx 0.7$. There, lattice momenta $\hat{p}_{\mu}^{2}=(2 / a)^{2} \sin ^{2}\left(a p_{\mu} / 2\right)$ differ from continuum ones by a few percent. In fact, for the standard discretisation (Wilson flow and plaquette discretisation for $E(x, t))$ one obtains

$$
\frac{\mathscr{E}_{\mathrm{Iat}}(t)}{\mathscr{E}(t)}=8\left(t / a^{2}\right)^{2} \pi^{2}\left\{\mathrm{e}^{-4 t / a^{2}} I_{0}\left(4 t / a^{2}\right)\right\}^{4}=1+k_{1} \frac{a^{2}}{t}+\ldots
$$

with $k_{1} \approx 0.13$ and small higher order terms for, say, $a^{2} / t \leq 1 / 2$. Similarly, $\left|t \mathscr{E}_{\text {lat }}^{\prime}(t) / \mathscr{E}(t)\right| \approx 0.12 \frac{a^{2}}{t}$ in the same range, where we normalise to $|\mathscr{E}(t)|$ since $\mathscr{E} \prime$ vanishes in the continuum limit at this order in the coupling. Obviously such lowest order perturbation theory estimates are just indicative.

While these are no particularly large cutoff effects, one may be interested in reducing them by Symanzik improvement and indeed, the Symanzik flow has been used in $[33,34]$. "Symanzik flow" refers to the flow equation, continuous in $t$, but with the gradient on the rhs. of the equation taken as the gradient of the tree-level Symanzik improved gauge action, while "Wilson flow" refers to the gradient of the Wilson plaquette action. Since the flow equation is a classical equation, without any explicit coupling constant, it is $\mathrm{O}\left(a^{2}\right)$ improved by using the tree level Symanzik improved action. However, this is not at all sufficient for full $\mathrm{O}\left(a^{2}\right)$ improvement. For full improvement, the following ingredients would be necessary:

- Symanzik flow, i.e. improvement of the (classical) flow equation.

- Improvement (classical) for the discretisation of $E(x, t)$.

- Improvement (with $g_{0}^{2}$ dependent coefficients) at the $t=0$ boundary.

- Improvement (with $g_{0}^{2}$ dependent coefficients) of the 4-d action.

This discussion uses the 5-d path integral (with Lagrange multiplier fields) [31, 32] and improvement of a theory with boundaries as previously developed for the Schrödinger functional. While the first two requirements are "easily" done by switching to the Symanzik flow and the Symanzik action density for $E(x, t)$, there are many terms for the other two. For example at the boundary one can have $t$-derivatives. With a dimension four field $\mathscr{O}$, the term $\left.a^{2} \int \mathrm{d}^{4} x \frac{\mathrm{d}}{\mathrm{d} t} \mathscr{O}(x, t)\right|_{t=0}$ is dimensionless and has to be considered as a term in the improved action. Systematic, non-perturbative, $\mathrm{O}\left(a^{2}\right)$ 
improvement seems out of reach! I discuss this here because after all the Symanzik flow is used in paractical computations.

Let me indicate the degree of improvement of the flow by (flow,discr), where flow is either "Sym" for the Symanzik flow or "Wils" the Wilson flow and discr is "plaq" for the plaquette discretisation of $E(x, t)$ or "clov" for the symmetric definition [30] formed from the clover $G_{\mu \nu}$. Boundary terms have so far not been considered. I therefore do not include them in my notation. Furthermore the degree of improvement of the 4-d action is indicated separately.

Incomplete improvement may be worse than no improvement. An example has been seen by A. Ramos in the leading order perturbative computation of $\mathscr{E}(x, t)$ in a Schrödinger functional setting, with (Wils,clov) once evaluated with the tree level improved 4-d action and once with the Wilson action. Improving just the 4-d gauge action leads to a factor $\approx 2$ increase of the cutoff effects in this case at leading order in the coupling.

I note that in Martin Lüscher's original pure gauge theory demonstration the difference of (Wils,clov) and (Wils,plaq) amounted to $\approx 6 \%$ in $t_{0} / r_{0}^{2}$ at $a=0.1 \mathrm{fm}$. This is the same magnitude as the estimate eq. (3.10).

Given the difficulties in systematically also removing $a^{2}$ terms, I think it is worth to consider to define a scale $t_{1}$ simply at somewhat larger flow time. In particular,

$$
\mathscr{E}\left(t_{1}\right)=2 / 3
$$

seems a good choice. At $t_{1}$ the kinematical cutoff effects eq. (3.10) are about a factor two suppressed (and better approximated by just $k_{1} a^{2} / t$ ) and the same would be the case for an analogous $w_{1}$. Obviously, $t_{1}$ will, however, be more sensitive to finite volume effects, which needs to be investigated.

\subsubsection{Precision}

A tremendous advantage of $t_{0}$ and $w_{0}$ is the high statistical precision. The variance of these quantities is very small and I note that due to the finiteness of correlation functions at all distances, it also remains finite in the continuum limit. On the other hand, at finite $t$ the autocorrelations are very much enhanced, see $[35,34,16]$. Despite this, the variance is so small that with a run of length $t_{\mathrm{MC}} / \tau_{\text {int }}\left(t_{0}\right) \approx t_{\mathrm{MC}} / \tau_{\exp }=20$ one achieves a precision of $\sqrt{t_{0}} / a$ at the level of around one per mille on a lattice of $3^{3} \times 6 \mathrm{fm}^{4}$. On larger lattices self averaging improves the precision further.

A second advantage of these scales is that $\mathscr{E}(t)$ is obtained as a straight expectation value. One does not need to extract the large time decay of some correlation function as it is the case for masses or $r_{0}, r_{1}$. Since the integration of the flow equation can be done with very high numerical precision, there is no systematic error - apart from the unavoidable discretisation and finite volume effects.

And finally, there is a weak quark mass dependence. The small quark mass expansion of expectation values of "local" fields, at fixed $t$, can be computed in chiral perturbation theory [29]. The NNLO formula for $\mathscr{E}(t)$ has been worked out by O. Bär and M. Golterman [36], with the interesting result that at and including order $m_{\pi}^{2}$ there are no non-analytic terms. This is in qualtitative agreement with the very linear mass-dependence seen in the chiral extrapolations, for example Fig. 4, below. 


\begin{tabular}{|c|c|c|c|c|c|c|c|c|c|c|}
\hline \multicolumn{3}{|c|}{ Wilson, $N_{\mathrm{f}}=2$} & \multicolumn{3}{|c|}{$\operatorname{tmQCD}, N_{\mathrm{f}}=2$} & \multicolumn{4}{|c|}{$N_{\mathrm{f}}>2$} & \multirow[b]{2}{*}{ from } \\
\hline$r_{0}[\mathrm{fm}]$ & & $\mathrm{m}$ & $r_{0}[\mathrm{fm}]$ & & $\mathrm{m}$ & $N_{\mathrm{f}}$ & $r_{0}[\mathrm{fm}]$ & $r_{1}[\mathrm{fm}]$ & & \\
\hline $0.503(10)$ & $f_{\mathrm{K}}$ & [14] & $0.438(14)$ & $f_{\mathrm{K}}$ & [38] & $2+1$ & $0.466(4)^{a}$ & $0.313(2)$ & div. & [39] \\
\hline $0.491(6)^{c}$ & $f_{\mathrm{K}}$ & [9] & & & & $2+1$ & & $0.321(5)$ & $\Upsilon$ & [1] \\
\hline $0.485(9)^{c}$ & $f_{\pi}$ & [9] & $0.420(20)$ & $f_{\pi}$ & [40] & $2+1$ & $0.470(4)$ & $0.311(2)$ & $f_{\pi}$ & {$[41,42]$} \\
\hline $0.501(15)^{b}$ & $m_{\mathrm{p}}$ & [43] & $0.465(16)$ & $m_{\mathrm{p}}$ & [44] & $2+1$ & $0.492(10)^{b}$ & & $m_{\Omega}$ & {$[11]$} \\
\hline \multirow[t]{2}{*}{$0.471(17)$} & $m_{\Omega}$ & [13] & & & & $2+1$ & $0.480(11)$ & $0.323(9)$ & $m_{\Omega}$ & {$[10]$} \\
\hline & & & & & & $2+1+1$ & & $0.311(3)$ & $f_{\pi}$ & [45] \\
\hline with $r_{0} / r_{1}$ anc & $/ a \mathrm{fr}$ & m [46] & & & ninary, & t this con & rence & & & \\
\hline
\end{tabular}

Table 1: Values for $r_{0}, r_{1}$. Column "from" shows the phenomenological scale used. All results except for those marked with ${ }^{b}$ have been obtained by a continuum extrapolation.

\section{Status}

I now want to give an impression how well we know the various scales at present. I will rather uncritically cite numbers from the literature and I would like to apologise that it is impossible to do justice to all computations. I tried to take into account the more recent numbers from the larger simulations.

\section{1 $m_{\Omega}$ and $m_{\mathrm{p}}$}

The masses of the Omega and the proton are experimentally known, so the relevant question is what kind of precision we are able to achieve in lattice computations. I neglect here the issue of the systematic uncertainty in quark mass extra/interpolations, even though this is rather relevant. I just note that precisions quoted for $m_{\Omega}$ (usually after the extrapolation to the physical point) are $\approx 0.5$ $\%$ [11], $\approx 1 \%$ [10], $\approx 1 \%$ [37], $\approx 5 \%$ [13]. Obviously, such numbers depend on the statistics. Nowadays it is typically around 1000 to 10000 molecular dynamics units. But they also depend on the starting value of a fit to a plateau, with an associated systematic error which is not so easily quantified.

\section{$4.2 r_{0}$ and $r_{1}$}

In large scale simulations, $r_{1} / a$ has been determined by MILC, HPQCD, RBC/UKQCD and HOTQCD; it has been converted to physical units for $N_{\mathrm{f}}=2+1$ using a variety of phenomenology scales, see Table 1 . Also modern determinations of $r_{0}$ are listed in that table.

There is still a considerable spread, most notably in the $N_{\mathrm{f}}=2$ theory for $r_{0}$ between the ALPHA and ETM collaborations. As the numbers refer mostly to the continuum limit they should agree. In Sect. 5 I return to the worrysome difference seen in Table 1.

For $N_{\mathrm{f}}>2$ all determinations performing a continuum extrapolation are compatible with

$$
r_{0}=0.472(5) \mathrm{fm}, \quad r_{1}=0.312(3) \mathrm{fm}
$$

I refrain from performing a weighted average of the numbers in Table 1 for the following reasons. Systematic errors, e.g. due to chiral extrapolations, may be relevant. More importantly, these 

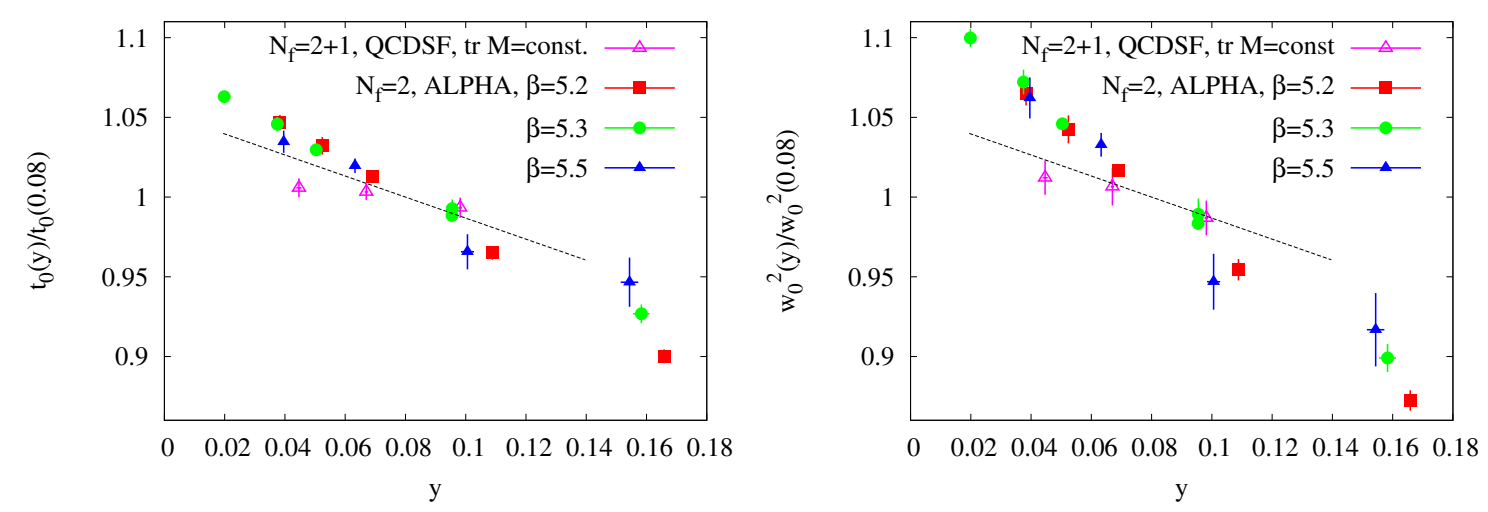

Figure 4: Mass dependence of the flow scales. We consider the functions $t_{0}(y), w_{0}(y)$ with the dimensionless measure $y=t_{0} m_{\pi}^{2}$ for the quark mass and normalised at the point $y=0.08$. Filled symbols are for $N_{\mathrm{f}}=2 \mathrm{CLS}$ lattices with $a=0.045 \mathrm{fm} \ldots 0.07 \mathrm{fm}$ [35] and open symbols are preliminary results by QCDSF for $N_{\mathrm{f}}=2+1$ along a trajectory $\operatorname{tr} m_{\text {quark }}=$ constant [47]. As a reference the dotted line correspond approximately to the behaviour $r_{0}^{2}$.

numbers are entirely dependent on a single set of simulations / action, the MILC configurations with rooted staggered fermions. There is only a weak (due to the much larger error) cross check by the RBC/UKQCD result $r_{0}=0.480(11) \mathrm{fm}, r_{1}=0.323(9) \mathrm{fm} \mathrm{[10]}$.

\section{$4.3 t_{0}$ and $w_{0}$}

Clearly, the newcomers $t_{0}$ and $w_{0}$ are most interesting. I discuss them in some more detail. In Fig. 4 I show the mass dependence for $N_{\mathrm{f}}=2$ and with open symbols the dependence in the $2+1$ theory along a trajectory with constant trace of the mass matrix. The results are normalised at an intermediate mass point defined by the variable $y=t_{0} m_{\pi}^{2}$, where $t_{0}$ is the mass-dependent quantity. The figures show first of all excellent scaling of the quark mass dependence of the $N_{\mathrm{f}}=2$ results with an $\mathrm{O}(a)$ improved action. Second a remarkably linear dependence is seen and third the comparison to the dotted line referring to $r_{0}^{2}(y) / r_{0}^{2}(0.08)$ indicates that $t_{0}$ has a somewhat stronger quark mass dependence than $r_{0}^{2}$ while $w_{0}^{2}$ has a significantly stronger one. As expected (see e.g.

\begin{tabular}{llll}
$N_{\mathrm{f}}$ & $\sqrt{t_{0}}[\mathrm{fm}]$ & $w_{0}[\mathrm{fm}]$ & from \\
\hline 0 & $0.1638(10)$ & $0.1670(10)$ & $r_{0}=0.49 \mathrm{fm}[35,30]$ \\
2 & $0.1539(12)$ & $0.1760(13)$ & $f_{\mathrm{K}}[35,9]$ \\
3 & $0.153(7)$ & $0.179(6)$ & $m_{\mathrm{p}}[47]$ \\
3 & $0.1465(25)$ & $0.1755(18)$ & $m_{\Omega}[33]$ \\
4 & $0.1420(8)$ & $0.1715(9)$ & $f_{\pi}[45]$ \\
4 & & $0.1712(6)$ & $f_{\pi}[34]$ \\
\hline
\end{tabular}

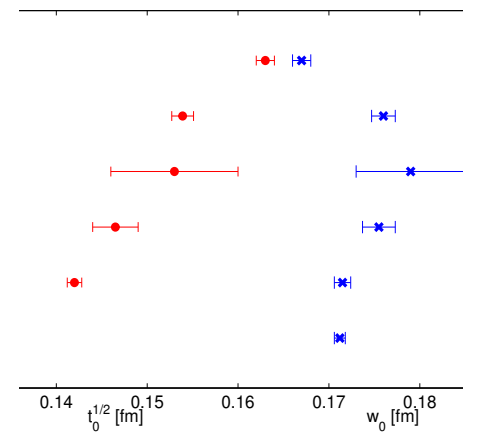

Table 2: Scales from the gradient flow. Note that these depend in principle on the phenomenological scale they are determined from. 
[36], the mass dependence along the trajectory $\operatorname{tr} m_{\text {quark }}=$ constant is quite a bit weaker. Rough estimates of the slopes are reported in Table 3 and $t_{0}, w_{0}$ in physical units in Table 2.

I have explained earlier that discretisation errors are a relevant, but also a subtle subject. General statements are difficult because discretisation errors are only meaningful after the scale has been set, or equivalently for dimensionless ratios. The same is true for the $N_{\mathrm{f}}$-dependence.

\begin{tabular}{llll|llllll}
$N_{\mathrm{f}}$ & $S_{t_{0}}^{\mathrm{m}}$ & $S_{w_{0}^{2}}^{\mathrm{m}}$ & Ref. & $N_{\mathrm{f}}$ & $S_{t_{0} / Q}^{\mathrm{a}}$ & $S_{w_{0}^{2} / Q}^{\mathrm{a}}$ & $Q$ & flow & Ref. \\
\hline & & & & 0 & $-1 \%$ & $-3 \%$ & $r_{0}^{2}$ & Wils & {$[30]$} \\
2 & $-12 \%$ & $-20 \%$ & {$[35]$} & 2 & $-8 \%$ & $-19 \%$ & $r_{0}^{2}$ & Wils & {$[35]$} \\
$2+1$ & & $-18 \%$ & {$[33]$} & $2+1$ & $-19 \%$ & $\approx 0$ & $m_{\Omega}^{-2}$ & Sym & {$[33]$} \\
$2+1+1$ & & $-13 \%$ & {$[34]$} & $2+1+1$ & & $\approx 0$ & $f_{\pi}^{-2}$ & Sym & {$[34]$} \\
& & & & $2+1+1$ & $7 \%$ & $\approx 0$ & $f_{\pi}^{-2}$ & Wils & {$[48,49]$}
\end{tabular}

Table 3: Slopes with respect to the mass, $S_{R}^{\mathrm{m}}$, eq. (1.5) and with respect to the lattice spacing, $S_{R}^{\mathrm{a}}=$

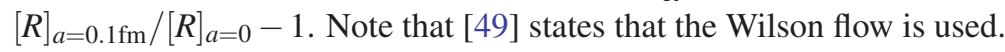

\subsection{Comparison of scales and $N_{\mathrm{f}}$-dependence.}

In Fig. 5, taken from [35] I show the approach to the limit $a \rightarrow 0$ of ratios of the scales $t_{0}, w_{0}^{2}, r_{0}^{2}$. Here $t_{0} / r_{0}^{2}$ has the smallest discretisation effects, while in [33], BMW reports small $a$-effects for $w_{0} m_{\Omega}$, compare for the slopes $S^{a}$ in Table 3 . Whether $t_{0}$ or $w_{0}$ have smaller cutoff effects depends on the details of the discretisation and/or the reference scale. General statements are not possible.

The Figure also shows a comparison with results for different $N_{\mathrm{f}}$. Here $[33,30,34,10,41$, 42, 39] enter, see [35]. The ratios demonstrate that the $N_{\mathrm{f}}=0$ and the $N_{\mathrm{f}}=2$ theories differ quite strongly for these purely gluonic infrared-dominated, non-perturbative, observables. The effects of the heavier quarks, strange and charm, appear to be less pronounced, but still noticeable. Of course, for a very heavy quark, decoupling is expected in such ratios. The charm quark may be heavy enough for decoupling to apply semiquantitatively.

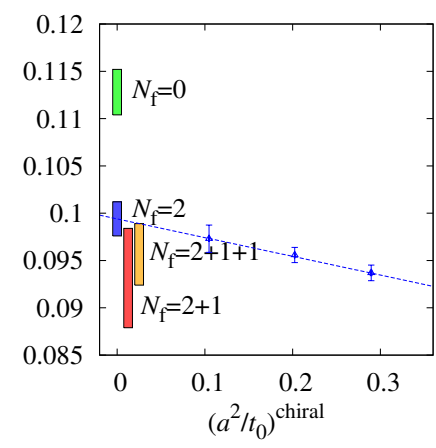

(a) $t_{0} / r_{0}^{2}$

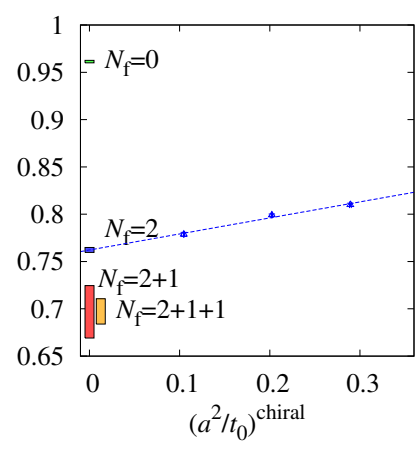

(b) $t_{0} / w_{0}^{2}$

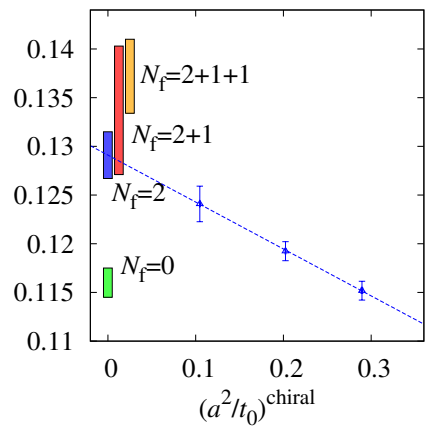

(c) $w_{0}^{2} / r_{0}^{2}$

Figure 5: Continuum extrapolation and flavour number dependence of ratios of scales. Taken from [35]. 

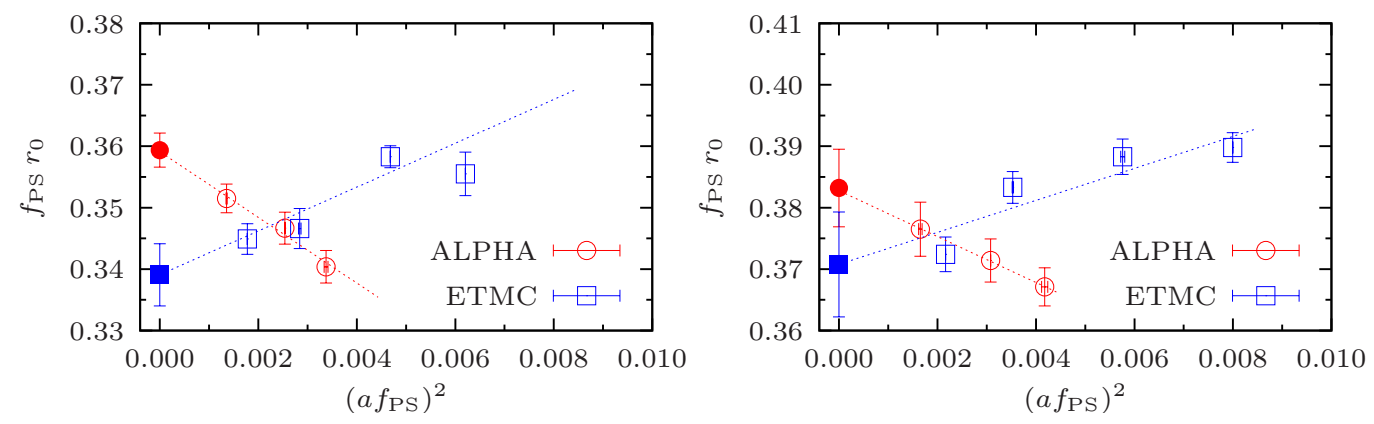

Figure 6: Continuum limit of $f_{\pi} r_{0}$ interpolated to $\left(m_{\pi} * r_{0}\right)^{2}=0.64$ (left) and to $\left(m_{\pi} * r_{0}\right)^{2}=1.128$ (right) for $N_{\mathrm{f}}=2$.

A similar $N_{\mathrm{f}}$-dependence is present for the ratio $r_{1} / r_{0}$ : It is about 0.66 for $N_{\mathrm{f}}=2+1[42,10]$, for $N_{\mathrm{f}}=2$ it has not directly been computed but from [27, 50] one can read off $r_{1} / r_{0} \approx 0.67$ and finally for $N_{\mathrm{f}}=0$ the results of [21] and others imply $r_{1} / r_{0}=0.73$.

At least part of the theory scales in physical units have to depend significantly on the number of flavours since an $N_{\mathrm{f}}$-dependence is present in the ratios. Note that in Table 2 the phenomenological reference scale(s) are mostly the light pseudo scalar decay constants, our best phenomenological scales at present. So mostly we are looking at a common reference scale.

\section{Open questions}

The considered theory scales are not listed in the particle data book. We have to determine them without prior knowledge. They therefore also constitute a good cross-check of our calculations as we should agree on their values, at least given the same $N_{\mathrm{f}}$ and experimental input scales. Unfortuantely for $N_{\mathrm{f}}=2$ the agreement on $r_{0}$ is not very good.

I therefore briefly discuss the differences in the determinations by the ALPHA and ETM collaborations. Comparing $r_{0}$ at the physical point, as is done in Table 1 , the origin of differences may be due to various sources, where, barring the possibility of actual mistakes, two particular ones come to mind. The first is the chiral extrapolation and the second is finite size effects. In order to see whether prior to any extrapolation, the two computations/discretisations do agree, G. Herdoiza performed an interpolation of dimensionless combinations $r_{0} f_{\pi}$ to two different reference pion masses, defined by $m_{\pi}^{2} r_{0}^{2}=0.640,1.128$. S. Lottini and B. Leder added the ALPHA collaboration points with a proper correlated error analysis. The surprising outcome, Fig. 6, is that the data actually agree reasonably well at intermediate lattice spacings, but the standard continuum extrapolations cross and lead to a few sigma difference at $a=0$ at the smaller pion mass. My attempt to understand this in terms of the finite $a$ chiral perturbation theory formulae for tmQCD of Oliver Bär [51] failed. In fact the formulae suggest larger cutoff effects than the data show, when $m_{\pi^{+}}^{2}-m_{\pi^{0}}^{2}$ from [52] is inserted. Maybe this suggests that the mass-splitting is actually smaller. In any case, the differences remain unexplained at present. As a speculation I add that there would be very good agreement if one just took the ETMC data at larger lattice spacings together with a very flat continuum extrapolation.

More work on this issue seems warrented. At least the analysis should be repeated with $r_{0} \rightarrow$ $\sqrt{t_{0}}$ in order to remove the possibility that there is something wrong with a determination of $r_{0}$. 
The present status provokes the questions: How well do we control our computations? Is our data ready for a continuum extrapolation?

\section{Conclusions}

This talk was scheduled at a time of transition. The all-time favorite $r_{0}, r_{1}$ are being replaced by scales derived from the gradient flow. The advantages of the latter are predominantly:

- There is an excellent statistical precision. Just 20 independent configurations lead to around per mille precision in the lattice spacing (of course the error is rather uncertain with only 20) when the lattice volume is reasonably large.

- Systematic errors due to excited state contributions are entirely absent in $\mathscr{E}(t)$ and consequently, as remarked in the question session at the conference, it is easy to compute $t_{0}, w_{0}$. There are no pitfalls in determining such scales.

In my opinion the second point is very important. The possible danger of a misidentified plateau is not immediately evident in Fig. 1. But for the example of the potential at large distances, where string breaking occurs, it has been seen that one has to be very careful in the selection of the right correlation function to find the correct ground state energy. More trivially, one may easily select a plateau value which is a sigma or two away from the true one because the errors at larger time mask the continuing variation of the effective mass. Only with plateaux as long as they are seen in the pseudo scalar sector one feels really safe.

The numbers listed in Table 2 do largely come from a preliminary analysis for this conference. They should better be reviewed again in one or two years time.

I think that it may be an improvement to define a scale at lower momenta; $t_{1}$, eq. (3.11) seems promising. It will be less sensitive to the details of the definition of the flow equation (concerning $a^{2}$ terms). Furthermore, a bit of reflection on the reported size of mass- and $N_{\mathrm{f}}$-dependence leads to the conclusion that both of these will be rather small for $t_{1}$. These advantages should be weighed in comparison to an expected increase in finite size effects which remain to be evaluated.

Finally I hope that the community will not leave the problem of Sect. 5 behind, but will solve it soon. Interesting physics results have been obtained with the scale from $f_{\pi}$ and $r_{0}$; some of them are certainly affected.

\section{Acknowledgments}

I would like to thank Martin Lüscher for very useful discussions before and after my presentation as well as for sharing his $N_{\mathrm{f}}=0$ data for the flow observables. I profited from work of the ALPHA collaboration, in particular by Mattia Bruno and Stefano Lottini. I thank Oliver Bär, Nathan Brown, Michele Della Morte, Albert Deuzeman, Gregorio Herdoiza, Georg von Hippel, Roger Horsley, Benjamin Jaeger, Björn Leder, Harvey Meyer, Alberto Ramos, Stefan Schaefer, Carsten Urbach for early communication of not yet published material and stimulating discussions. Many thanks go to Tom de Grand for relevant questions on the talk and Ulli Wolff for a critical reading of the manuscript. I acknowledge support by the Deutsche Forschungsgemeinschaft (SFB/TR 9) and the European Community (grant 283826, HadronPhysics3). 


\section{References}

[1] HPQCD Collaboration, UKQCD Collaboration Collaboration, E. Follana, C. Davies, G. Lepage, and J. Shigemitsu, High Precision determination of the pi, $K, D$ and $D(s)$ decay constants from lattice QCD, Phys.Rev.Lett. 100 (2008) 062002, [arXiv:0706.1726].

[2] C. Davies, C. McNeile, E. Follana, G. Lepage, H. Na, et al., Update: Precision $D_{s}$ decay constant from full lattice QCD using very fine lattices, Phys.Rev. D82 (2010) 114504, [arXiv: 1008 . 4018 ].

[3] QCDSF/UKQCD Collaboration Collaboration, M. Göckeler et al., Determination of light and strange quark masses from full lattice QCD, Phys.Lett. B639 (2006) 307-311, [hep-ph / 0409312 ].

[4] A. Cooke, R. Horsley, Y. Nakamura, D. Pleiter, P. Rakow, et al., SU(3) flavour breaking and baryon structure, arXiv:1311.4916.

[5] ALPHA Collaboration, U. Wolff, Monte carlo errors with less errors, Comput. Phys. Commun. 156 (2004) 143-153, [hep-lat/0306017].

[6] R. Sommer, A new way to set the energy scale in lattice gauge theories and its applications to the static force and $\alpha_{s}$ in SU(2) Yang-Mills theory, Nucl. Phys. B411 (1994) 839, [hep-lat/9310 022 ].

[7] G. P. Lepage, The analysis of algorithms for lattice field theory, . in From Actions to Answers, edited by T. DeGrand and D. Toussaint (World Scientic, Singapore, 1989).

[8] M. Lüscher, Computational Strategies in Lattice QCD, arXiv:1002 .4232. Lecture note of the Summer School on "Modern perspectives in lattice QCD”, Les Houches, August 3-28, 2009.

[9] S. Lottini, Chiral behaviour of the pion decay constant in $N_{f}=2$ QCD, arXiv:1311.3081.

[10] RBC Collaboration, UKQCD Collaboration Collaboration, R. Arthur et al., Domain Wall QCD with Near-Physical Pions, Phys.Rev. D87 (2013) 094514, [arXiv: 1208 . 4412].

[11] PACS-CS Collaboration Collaboration, S. Aoki et al., Physical Point Simulation in 2+1 Flavor Lattice QCD, Phys.Rev. D81 (2010) 074503, [arXiv:0911.2561].

[12] B. Jäger, T. Rae, S. Capitani, M. Della Morte, D. Djukanovic, et al., A high-statistics study of the nucleon EM form factors, axial charge and quark momentum fraction, arXiv: 1311.5804.

[13] S. Capitani, M. Della Morte, G. von Hippel, B. Knippschild, and H. Wittig, Scale setting via the $\Omega$ baryon mass, PoS LATTICE2011 (2011) 145, [arXiv: 1110 . 6365].

[14] P. Fritzsch, F. Knechtli, B. Leder, M. Marinkovic, S. Schaefer, et al., The strange quark mass and Lambda parameter of two flavor QCD, Nucl.Phys. B865 (2012) 397-429, [arXiv: 1205.5380 ].

[15] ALPHA Collaboration Collaboration, S. Schaefer, R. Sommer, and F. Virotta, Critical slowing down and error analysis in lattice QCD simulations, Nucl.Phys. B845 (2011) 93-119, [arXiv:1009.5228].

[16] M. Lüscher and S. Schaefer, Lattice QCD without topology barriers, JHEP 1107 (2011) 036, [arXiv:1105.4749].

[17] MILC collaboration Collaboration, A. Bazavov et al., Topological susceptibility with the asqtad action, Phys.Rev. D81 (2010) 114501, [arXiv: 1003 .5695].

[18] F. Jegerlehner and A. Nyffeler, The Muon g-2, Phys.Rept. 477 (2009) 1-110, [arXiv: 0902.3360 ].

[19] D. Bernecker and H. B. Meyer, Vector Correlators in Lattice QCD: Methods and applications, Eur.Phys.J. A47 (2011) 148, [arXiv: 1107.4388 ]. 
[20] A. Francis, G. von Hippel, F. Jegerlehner, and H. B. Meyer, Vector correlator and scale determination in lattice $Q C D$, arXiv:1312.0035.

[21] S. Necco and R. Sommer, The $N(f)=0$ heavy quark potential from short to intermediate distances, Nucl. Phys. B622 (2002) 328-346, [http: / / arXiv.org/abs/hep-lat/ 0108008 ].

[22] ALPHA Collaboration, M. Kurth and R. Sommer, Renormalization and $O(a)$-improvement of the static axial current, Nucl. Phys. B597 (2001) 488-518, [hep-lat/ 0007002$].$

[23] A. Hasenfratz and F. Knechtli, Flavor symmetry and the static potential with hypercubic blocking, Phys. Rev. D64 (2001) 034504, [hep-lat/ 0103029 ].

[24] M. Della Morte, A. Shindler, and R. Sommer, On lattice actions for static quarks, JHEP 0508 (2005) 051, [hep-lat/0506008].

[25] M. Lüscher, P. Weisz, and U. Wolff, A numerical method to compute the running coupling in asymptotically free theories, Nucl. Phys. B359 (1991) 221-243.

[26] B. Blossier, M. Della Morte, G. von Hippel, T. Mendes, and R. Sommer, On the generalized eigenvalue method for energies and matrix elements in lattice field theory, JHEP 04 (2009) 094, [arXiv:0902.1265].

[27] M. Donnellan, F. Knechtli, B. Leder, and R. Sommer, Determination of the Static Potential with Dynamical Fermions, Nucl.Phys. B849 (2011) 45-63, [arXiv: 1012 . 3037].

[28] C. W. Bernard, T. Burch, K. Orginos, D. Toussaint, T. A. DeGrand, et al., The Static quark potential in three flavor QCD, Phys.Rev. D62 (2000) 034503, [hep-lat/ 0002028 ].

[29] M. Lüscher, Future applications of the Yang-Mills gradient flow in lattice QCD, arXiv:1308.5598.

[30] M. Lüscher, Properties and uses of the Wilson flow in lattice QCD, JHEP 1008 (2010) 071, [arXiv:1006.4518].

[31] M. Lüscher and P. Weisz, Perturbative analysis of the gradient flow in non-abelian gauge theories, JHEP 1102 (2011) 051, [arXiv: 1101.0963$].$

[32] M. Lüscher, Chiral symmetry and the Yang-Mills gradient flow, JHEP 1304 (2013) 123, [arXiv:1302.5246].

[33] S. Borsanyi, S. Durr, Z. Fodor, C. Hoelbling, S. D. Katz, et al., High-precision scale setting in lattice QCD, JHEP 1209 (2012) 010, [arXiv: 1203.4469].

[34] The MILC Collaboration Collaboration, A. Bazavov et al., Symanzik flow on HISQ ensembles, arXiv: 1311.1474.

[35] M. Bruno and R. Sommer, On the $N_{f}$-dependence of gluonic observables, arXiv:1311.5585.

[36] O. Bär and M. Golterman, Chiral perturbation theory for gradient flow observables, arXiv:1312.4999.

[37] S. Dürr, Z. Fodor, C. Hoelbling, S. Katz, S. Krieg, et al., Lattice QCD at the physical point: Simulation and analysis details, JHEP 1108 (2011) 148, [arXiv:1011.2711].

[38] [ETM 09], B. Blossier, et al., Pseudoscalar decay constants of kaon and D-mesons from $N_{f}=2$ twisted mass Lattice QCD, JHEP 0907 (2009) 043, [arXiv: 0904.0954 ].

[39] [HPQCD 09B], C. Davies, E. Follana, I. Kendall, G. P. Lepage, and C. McNeile, Precise determination of the lattice spacing in full lattice QCD, Phys.Rev. D81 (2010) 034506, [arXiv:0910.1229]. 
[40] [ETM 09C], R. Baron, et al., Light meson physics from maximally twisted mass lattice QCD, JHEP 08 (2010) 097, [arXiv:0911.5061].

[41] [MILC 10], A. Bazavov, et al., Results for light pseudoscalar mesons, PoS LAT2010 (2010) 074, [arXiv:1012.0868].

[42] A. Bazavov, T. Bhattacharya, M. Cheng, C. DeTar, H. Ding, et al., The chiral and deconfinement aspects of the QCD transition, Phys.Rev. D85 (2012) 054503, [arXiv: 1111.1710].

[43] G. Bali, P. Bruns, S. Collins, M. Deka, B. Glasle, et al., Nucleon mass and sigma term from lattice QCD with two light fermion flavors, Nucl.Phys. B866 (2013) 1-25, [arXiv:1206. 7034].

[44] ETM Collaboration Collaboration, C. Alexandrou et al., Low-lying baryon spectrum with two dynamical twisted mass fermions, Phys.Rev. D80 (2009) 114503, [arXiv: 0910.2419 ].

[45] R. Dowdall, C. Davies, G. Lepage, and C. McNeile, Vus from pi and K decay constants in full lattice QCD with physical $u, d$, s and c quarks, Phys.Rev. D88 (2013) 074504, [arXiv:1303.1670].

[46] A. Bazavov, D. Toussaint, C. Bernard, J. Laiho, C. DeTar, et al., Nonperturbative QCD simulations with 2+1 flavors of improved staggered quarks, Rev.Mod.Phys. 82 (2010) 1349-1417, [arXiv:0903.3598].

[47] R. Horsley, J. Najjar, Y. Nakamura, H. Perlt, D. Pleiter, et al., SU(3) flavour symmetry breaking and charmed states, arXiv:1311.5010.

[48] R. Dowdall, C. Davies, G. Lepage, and C. McNeile, Vus from pi and K decay constants in full lattice QCD with physical $u, d$, s and c quarks, Phys.Rev. D88 (2013) 074504, [arXiv:1303.1670].

[49] R. Dowdall, C. Davies, R. Horgan, G. Lepage, C. McNeile, et al., B, Bs, K and pi weak matrix elements with physical light quarks, . Here it is made explicit that the Wilson flow is used.

[50] F. Knechtli and B. Leder, The shape of the static potential with dynamical fermions, arXiv: 1112.1246.

[51] O. Bär, Chiral logs in twisted mass lattice QCD with large isospin breaking, Phys.Rev. D82 (2010) 094505, [arXiv: 1008.0784$]$.

[52] G. Herdoiza, K. Jansen, C. Michael, K. Ottnad, and C. Urbach, Determination of Low-Energy Constants of Wilson Chiral Perturbation Theory, JHEP 1305 (2013) 038, [arXiv: 1303.3516 ]. 